\title{
STABILITY ANALYSIS OF NONLINEAR LYAPUNOV SYSTEMS ASSOCIATED WITH AN $n$ TH ORDER SYSTEM OF MATRIX DIFFERENTIAL EQUATIONS
}

\author{
K.N. MURTY ${ }^{1}$ and MICHAEL D. SHAW \\ Florida Institute of Technology \\ Department of Mathematical Sciences \\ Melbourne, FL 32901 USA
}

(Received March, 2000; Revised October, 2000)

\begin{abstract}
This paper introduces the notion of Lipschitz stability for nonlinear $n$th order matrix Lyapunov differential systems and gives sufficient conditions for Lipschitz stability. We develop variation of parameters formula for the solution of the nonhomogeneous nonlinear $n$th order matrix Lyapunov differential system. We study observability and controllability of a special system of $n$th order nonlinear Lyapunov systems.

Key words: Controllability, Lyapunov Systems, Matrix Differential Equations, Observability, Stability Analysis, Variation of Parameters.
\end{abstract}

AMS subject classifications: 34D10, 34D99.

\section{Introduction}

Stability analysis of nonlinear systems is an important area of current research and many concepts of stability analysis have been recently developed for first order nonlinear Lyapunov systems. In this paper, we develop a variation of parameters formula for $n$th order nonlinear Lyapunov systems and use it as a tool to study the various concepts of stability. Our paper is organized as follows: In Section 2, we develop the nonlinear variation of parameters formula for nonhomogeneous systems. In Section 3, we study various concepts of stability for homogeneous systems. In Section 4, we introduce the notion of Lipschitz stability and investigate sufficient conditions for the $n$th order nonlinear system to be Lipschitz stable and uniformly Lipschitz stable. In Section 5, we study the controllability and observability of the $n$th order nonlinear Lyapunov system.

\section{General Solution of Homogeneous Systems, Nonlinear Variation of Parameters}

In this section, we develop the general solution of the nonlinear matrix Lyapunov system

$$
T^{(n)}=A^{n} T(t)+n C_{1} A^{n-1} T(t) B+n C_{2} A^{n-2} T(t) B^{2}+\ldots
$$

\footnotetext{
${ }^{1}$ Permanent address: Andhra University, Department of Applied Mathematics, Viskhapatnam, India.
} 


$$
+n C_{r} A^{n-r} T(t) B^{r}+\ldots+T(t) B^{n}
$$

in terms of two fundamental matrix solutions of $T^{\prime}=A T(t)$ and $T^{\prime}=B^{*} T(t)$ where $B^{*}$ is the transpose of the complex conjugate matrix of $B$. In (2.1), $A$ and $B$ are constant square matrices of order $n \times n$ and $T \in C\left[\mathbb{R}_{+}, \mathbb{R}^{n} \times \mathbb{R}^{n}\right]$ is a variable matrix. Throughout this paper, $Y(t)$ and $Z(t)$ stand for the fundamental matrix solutions of $T^{\prime}=A T$ and $T^{\prime}=B^{*} T$.

Theorem 2.1: If $Y$ is a fundamental matrix solution of $T^{\prime}=A T$, then $Y^{(n)}$ is a fundamental matrix solution of $T^{\prime}=A^{n} T$ where $n$ is a positive integer.

Proof: $Y$ is a fundamental matrix solution of $T^{\prime}=A T$ if and only if $Y^{\prime}(t)=A Y(t)$. This implies that $Y^{\prime \prime}(t)=A Y^{\prime}(t)=A A Y=A^{2} Y$. This further implies that $Y^{\prime \prime}(t)=A^{3} Y$ and so on. Hence, $Y^{(n)}(t)=A^{n} Y(t)$. Thus, $Y$ is also a fundamental matrix solution of $T^{\prime}=A^{n} T$.

Theorem 2.2: Let $C$ be a constant square matrix of order $n \times n$. Then any solution of

$$
T^{(n)}=\sum_{r=0}^{n}\left(\begin{array}{l}
n \\
r
\end{array}\right) A^{n-r} T(t) B^{r}
$$

with $A^{0}=B^{0}=I$, is of the form $T(t)=Y(t) C Z^{*}(t)$, where $Y(t)$ is a fundamental matrix solution of $T^{\prime}=A T$ and $Z(t)$ is a fundamental matrix solution of $T^{\prime}=B^{*} T$.

Proof: It can be easily verified that $T$ defined by $T(t)=Y(t) C Z^{*}(t)$ is a solution of (2.2). For

$$
\begin{gathered}
\left(Y(t) C Z^{*}(t)\right)^{(n)}=A^{n} Y(t) C Z^{*}(t)+n C_{1} A^{n-1} Y(t) C Z^{*}(t) B \\
+n C_{2} A^{n-2} Y(t) C Z^{*}(t) B^{2}+\ldots+n C_{r} A^{n-r} Y(t) C Z^{*}(t) B^{r} \\
+\ldots+Y(t) C Z^{*}(t) B^{n} .
\end{gathered}
$$

By the Leibnitz theorem, we have

$$
\begin{gathered}
\left(Y(t) C Z^{*}(t)\right)^{(n)}=Y^{(n)}(t) C Z^{*}(t)+n C_{1} Y^{(n-1)}(t) C Z^{*^{\prime}}(t) \\
+n C_{2} Y^{(n-2)}(t) C Z^{*^{\prime \prime}}(t)+\ldots+n C_{r} Y^{(n-r)}(t) C Z^{*(r)}(t)+\ldots+Y(t) C Z^{*^{(n)}}(t) .
\end{gathered}
$$

The two equations are the same since $Y^{(k)}(t)=A^{k} Y(t)$ and $Z^{*^{(k)}}(t)=Z^{*}(t) B^{k}$ for $k=1,2, \ldots, n$. Thus, $T(t)=Y(t) C Z^{*}(t)$ is a solution of (2.1). To prove that every solution of the equation is of this form, let $T(t)$ be a solution of (2.2) and let $K$ be a square matrix of order $n \times n$ defined by $K(t)=Y^{-1}(t) T(t)$. Then $T(t)=Y(t) K(t)$. Now, $T(t)=Y(t) K(t)$ if and only if

$$
\begin{gathered}
Y^{(n)}(t) K(t)+n C_{1} Y(t)^{n-1} K^{\prime}(t)+n C_{2} Y(t)^{n-2} K^{\prime \prime}(t)+\ldots \\
+n C_{r} Y(t)^{n-r} K^{(r)}(t)+\ldots+Y(t) K^{(n)}(t) \\
=A^{n} Y(t) K(t)+n C_{1} A^{n-1} Y(t) K^{\prime}(t)+n C_{2} A^{n-2} Y(*)(t) K^{\prime \prime}(t)+\ldots \\
+n C_{r} A^{n-r} Y(t) K^{(r)}(t)+\ldots+Y(t) K^{(n)}(t) .
\end{gathered}
$$

Since $Y(t), Y^{\prime}(t), \ldots$, and $Y^{(n)}(t)$ are linearly independent, equation (2.3) can hold if and only if $K^{(k)}=K B^{k}$ if and only if $K^{*^{(k)}}=B^{* k} K^{*}$ for $k=1,2, \ldots, n$. Since $Z(t)$ is a fundamental matrix solution of $T^{\prime}=B^{*} T$, it follows that $Z$ also a fundamental 
matrix solution of $T^{(n)}=B^{*^{n}} T$. Therefore, there exists a constant matrix $C$ such that $K^{*}=Z C^{*}$, then $T=Y K=Y C Z^{*}$ and the proof is complete.

We shall now develop the variation of parameters formula for the solution of the nonhomogeneous system

$$
T^{(n)}=\sum_{r=0}^{n}\left(\begin{array}{l}
n \\
r
\end{array}\right) A^{n-r} T(t) B^{r}+F(t, T(t)) .
$$

Theorem 2.3: Any solution $T(t)$ of (2.4) is of the form $T(t)=Y(t) C Z^{*}(t)+T_{p}(t)$, where $T_{p}(t)$ is a particular solution of $(2.4)$.

Proof: It can easily be verified that $T(t)$ defined by $T(t)=Y(t) C Z^{*}(t)+T_{p}(t)$ is a solution of (2.4). To prove that every solution is of this form, let $T$ be any solution of (2.4) and $T_{p}(t)$ be any particular solution of (2.4). Then $T(t)-T_{p}(t)$ is a solution of the linear homogeneous system (2.2) and has the form $Y(t) C Z^{*}(t)$. Hence, $T(t)-T_{p}(t)=Y(t) C Z^{*}(t)$ and $T(t)=Y(t) C Z^{*}(t)+T_{p}(t)$. The proof is complete.

Theorem 2.4: Let $Y(t)$ be a fundamental matrix solution of $T^{\prime}=A T$ and $Z(t)$ be a fundamental matrix solution of $T^{\prime}=B^{*} T$. Furthermore, suppose that $C \in C^{n}\left[\mathbb{R}_{+}, \mathbb{R}^{n} \times \mathbb{R}^{n}\right]$ such that

$$
\left(Y^{\prime} C^{\prime} Z^{*}+Y C^{\prime} Z^{*^{\prime}}\right)^{(i-2)}=0 \text { for } i=1,2, \ldots, n .
$$

Then a particular solution $T_{p}(t)$ of (2.4) is given by

$T_{p}(t)=$

$$
Y(t)\left(\int_{a}^{t} \int_{a}^{\tau_{1}} \int_{a}^{\tau_{2}} \ldots \int_{a}^{\tau_{n-1}} Y^{-1}(s) F(s, T(s)) Z^{*^{-1}}(s) d s d \tau_{n-1} \ldots d \tau_{2} d \tau_{1}\right) Z^{*}(t) .
$$

Proof: Any solution of the homogeneous system (2.1) is of the form $T(t)=$ $Y(t) C Z^{*}(t)$, where $C$ is an $n \times n$ constant matrix. Such a solution cannot be a solution of the nonhomogeneous system (2.4) unless $F=0$. Assume $C \in$

$C^{n}\left[\mathbb{R}_{+}, \mathbb{R}^{n} \times \mathbb{R}^{n}\right]$ and seek a particular solution $T_{p}(t)$ of the form $T_{p}(t)=$ $Y(t) C(t) Z^{*}(t)$. Substituting $T_{p}(t)$ into (2.3) and using (2.5) yields $Y(t) C^{(n)}(t)$

$Z^{*}(t)=F(t, T(t))$ which in turn yields $C^{(n)}(t)=Y^{-1}(t) F(t, T(t)) Z^{*^{-1}}(t)$. After integrating $n$ times, we have

$$
C(t)=\int_{a}^{t} \int_{a}^{\tau_{1}} \int_{a}^{\tau_{2}} \ldots \int_{a}^{\tau_{n-1}} Y^{-1}(s) F(s, T(s)) Z^{*^{-1}}(s) d s d \tau_{n-1} \ldots d \tau_{2} d \tau_{1} .
$$

Hence,

$$
\begin{aligned}
T_{p}(t)= & \\
& Y(t)\left(\int_{a}^{t} \int_{a}^{\tau_{1}} \int_{a}^{\tau_{2}} \ldots \int_{a}^{\tau_{n-1}} Y^{-1}(s) F(s, T(s)) Z^{*^{-1}}(s) d s d \tau_{n-1} \ldots d \tau_{2} d \tau_{1}\right) Z^{*}(t)
\end{aligned}
$$

and $T_{p}(t)$ is easily verified as a solution of (2.4).

Theorem 2.5: Any solution $T(t)$ of the nonhomogeneous Lyapunov system (2.4) is of the form $T(t)=Y(t) C Z^{*}(t)+T_{p}(t)$ where $T_{p}(t)$ is given by $(2.6)$. 
Proof: For $n=1$, the homogeneous system (2.2) reduces to $T^{\prime}=A T+T B$. The general solution in terms of the variation of parameters formula for $T^{\prime}=A(t) T(t)+$ $T(t) B(t)+F(t, T(t))$ has been established in [4].

And for $n>1$, we have if $T(t)$ is any solution of (2.4) and $T_{p}(t)$ is a particular solution of (2.4), then $T(t)-T_{p}(t)$ is a solution of the homogeneous system (2.1). Hence, $T(t)-T_{p}(t)=Y(t) C Z^{*}(t)$ and $T(t)=Y(t) C Z^{*}(t)+T_{p}(t)$.

\section{Stability Analysis of the Homogeneous System}

In this section, we shall be concerned with the various concepts of stability of the homogeneous system

$$
T^{(n)}=\sum_{r=0}^{n}\left(\begin{array}{l}
n \\
r
\end{array}\right) A^{n-r} T(t) B^{r}
$$

The proofs of the next theorem in each case are simple and hence omitted. For basic results on stability, see [1].

Theorem 3.1: Let $Y(t)$ be a fundamental matrix solution of $T^{\prime}=A T$ and $Z(t)$ be a fundamental matrix solution of $T^{\prime}=B^{*} T$. Then the matrix system (3.1)

(a) is stable if and only if there exists a positive constant $K$ such that

$$
\|Y(t)\|\|Z(t)\| \leq K \text { for all } t \geq t_{0} \geq 0,
$$

(b) is asymptotically stable if and only if it is stable and

$$
\|Y(t)\|\|Z(t)\| \rightarrow 0 \text { as } t \rightarrow \infty
$$

(c) is uniformly stable if and only if there exists a positive constant $K$ such that

$$
\left\|Y(t) Y^{-1}(s)\right\|\left\|Z(t) Z^{-1}(s)\right\| \leq K \text { for all } t \geq s \geq t_{0} \geq 0,
$$

(d) is strongly stable if and only if there exists a positive constant $K$ such that

$$
\left\|Y(t) Y^{-1}(t)\right\|\left\|Z(t) Z^{-1}(t)\right\| \leq K \text { for all } t \geq t_{0} \geq 0 \text {, and }
$$

(e) is uniformly asymptotically stable if and only if there exist positive constants $m_{1}, m_{2}, \alpha$, and $\beta$ such that

$$
\left\|Y(t) Y^{-1}(s)\right\| \leq m_{1} e^{-\alpha(t-s)}
$$

and

$$
\left\|Z(t) Z^{-1}(s)\right\| \leq m_{2} e^{-\beta(t-s)} \text { for } t_{0} \leq s \leq t<\infty .
$$

Theorem 3.2: Suppose there exist positive constants $L_{1}$ and $L_{2}$ such that $\left\|Y(t) Y^{-1}(s)\right\| \leq L_{1}$ and $\left\|Z(t) Z^{-1}(s)\right\| \leq L_{2}$ for all $\infty>t \geq s \geq t_{0}$ and $F$ satisfies the condition $\|F(t, T(t))\| \leq \gamma(t)\|T(t)\|$ where $\gamma$ is a positive continuous function such that $\int_{t_{0}}^{\infty} \int_{t_{0}}^{\infty} \cdots \int_{t_{0}}^{\infty} \gamma(s) d s<\infty$. Then there exists a positive constant $L$ such that for any $t_{1} \geq t_{0}$ and $\left\|T\left(t_{1}\right)\right\| \leq c / L$, we have $\|T(t)\| \leq L\left\|T\left(t_{1}\right)\right\|$ for all $t \geq t_{1} \geq t_{0}$. 
Proof: Any solution $T(t)$ of the nonhomogeneous matrix Lyapunov system (2.4) satisfying $T\left(t_{1}\right)=T_{1}$ has the form

$$
\begin{gathered}
T(t)=Y(t) Y^{-1}\left(t_{1}\right) T\left(t_{1}\right) Z^{*^{-1}}\left(t_{1}\right) Z^{*}(t) \\
+Y(t)\left(\int_{t_{1}}^{t} \int_{t_{1}}^{\tau_{1}} \ldots \int_{t_{1}}^{\tau_{n-1}} Y^{-1}(s) F(s, T(s)) Z^{*^{-1}}(s) d s d \tau_{n-1} \ldots d \tau_{1}\right) Z^{*}(t) .
\end{gathered}
$$

Hence,

$$
\begin{gathered}
\|T(t)\| \leq\left\|Y(t)^{-1}\left(t_{1}\right)\right\|\left\|T\left(t_{1}\right)\right\|\left\|Z^{*^{-1}}\left(t_{1}\right) Z^{*}(t)\right\| \\
+\int_{t_{1}}^{t} \int_{t_{1}}^{\tau_{1}} \ldots \int_{t_{1}}^{\tau_{n-1}}\left\|Y(t) Y^{-1}(s)\right\|\|F(s, T(s))\|\left\|Z^{*^{-1}}(s) Z^{*}(t)\right\| d s d \tau_{n-1} \ldots d \tau_{1} \\
\leq L_{1} L_{2}\left\|T\left(t_{1}\right)\right\|+\int_{t_{1}}^{t} \int_{t_{1}}^{\tau_{1}} \ldots \int_{t_{1}}^{\tau_{n-1}} L_{1} L_{2} \gamma(s)\|T(s)\| d s d \tau_{n-1} \ldots d \tau_{1} \\
\leq L_{1} L_{2}\left\|T\left(t_{1}\right)\right\|+L_{1} L_{2} \int_{t_{1}}^{t} \int_{t_{1}}^{\tau_{1}} \ldots \int_{t_{1}}^{\tau_{n-1}} L_{1} L_{2} \gamma(s)\|T(s)\| d s d \tau_{n-1} \ldots d \tau_{1} .
\end{gathered}
$$

By applying the Reid-Bellman inequality [2], we obtain

$$
\begin{gathered}
\|T(t)\| \leq L_{1} L_{2}\left\|T\left(t_{1}\right)\right\| \exp \left(\int_{t_{1}}^{t} \int_{t_{1}}^{\tau_{1}} \ldots \int_{t_{1}}^{\tau_{n-1}} \gamma(s) d s d \tau_{n-1} \ldots d \tau_{1}\right) \\
\leq L\left\|T\left(t_{1}\right)\right\| \text { for } t_{1}<t<\infty
\end{gathered}
$$

Theorem 3.3: Suppose there exists an $m=\max \left\{m_{1}, m_{2}\right\}>0$ such that $\left\|Y(t) Y^{-1}(s)\right\| \leq m_{1} e^{-\alpha(t-s)}$ and $\left\|Z(t) Z^{-1}(s)\right\| \leq m_{2} e^{-\beta(t-s)}$ for $t_{0} \leq s \leq t<\infty$ where $\alpha$ and $\beta$ are constants such that $\alpha+\beta>0$ and $F$ satisfies a Lipschitz condition with the Lipschitz constant $L<C / m$. Then there exists a positive constant $K$ such that $\left\|T\left(t_{1}\right)\right\| \leq(\alpha+\beta) / K$ implies

$$
\begin{aligned}
& \|T(t)\| \leq m_{1} m_{2} \exp \left(-(\alpha+\beta) t_{1}\right)\left\|T\left(t_{1}\right)\right\| \\
& \times \exp \left(m_{1} m_{2} \int_{t_{1}}^{t} \int_{t_{1}}^{\tau_{1}} \ldots \int_{t_{1}}^{\tau_{n-1}} \gamma(s) d s d \tau_{n-1} \ldots d \tau_{1}\right) .
\end{aligned}
$$

Proof: The proof utilizes the variation of parameters formula given in Theorem 2.4.

\section{Lipschitz Stability of the Nonhomogeneous Lyapunov System}

In this section, we give sufficient conditions for Lipschitz stability on nonlinear $n$th order matrix Lyapunov differential systems and study Lipschitz stability properties of the zero solution of perturbed matrix Lyapunov system with the variation of parameters formula developed in Section 2. Before we proceed to give our new results, we shall give the following definitions [2, 3]. 
Definition 4.1: The zero solution of the $n$th order Lyapunov system (2.4) is said to be uniformly Lipschitz stable if there exists a constant $m>0$ and a $\delta>0$ such that

$$
\left\|T\left(t, t_{0}, T_{0}\right)\right\| \leq m\left\|T_{0}\right\| \text { whenever }\left\|T_{0}\right\| \leq \delta \text { and } t \geq t_{0} \geq 0
$$

Definition 4.2: The zero solution of the $n$th order Lyapunov system (2.4) is said to be asymptotically stable if there exist positive constants $m$ and $\alpha$ such that

$$
\left\|T\left(t, t_{0}, T_{0}\right)\right\| \leq m\left\|T_{0}\right\| e^{-\alpha\left(t-t_{0}\right)} \text { whenever }\left\|T_{0}\right\| \leq \delta \text { and } t \geq t_{0} \geq 0
$$

Let $f(t, T(t))=\sum_{r=0}^{n}\left(\begin{array}{l}n \\ r\end{array}\right) A^{n-r} T(t) B^{r}$ with $B^{0}=A^{0}=1$ and the linear system be

$$
T^{\prime}=f(t, T(t))
$$

and its perturbed system be

$$
W^{\prime}=f(t, W(t))+F(t, W(t))
$$

satisfying the initial data $T\left(t_{0}\right)=T_{0}$, where $t_{0} \in \mathbb{R}_{+}$and $f, F \in C\left[\mathbb{R}_{+} \times \mathbb{R}^{n} \times \mathbb{R}^{n}\right.$, $\left.\mathbb{R}^{n} \times \mathbb{R}^{n}\right]$. Assume that $Y(t)$ is a fundamental matrix solution of $T^{\prime}=A T$ and $Z(t)$ be a fundamental matrix solution of $T^{\prime}=B^{*} T$. Obviously, system (4.1) admits the zero solution. We now have the following theorems.

Theorem 4.1: Suppose that

$$
\begin{gathered}
\| Y^{-1}\left(t, t_{0}, T_{0}\right) F\left(t, T\left(t, t_{0}, T_{0}\right) \| \leq g\left(t,\left\|T_{0}\right\|\right)\right. \text { or } \\
\left\|F\left(t, T\left(t, t_{0}, T_{0}\right)\right) Z^{-1}\left(t, t_{0}, T_{0}\right)\right\| \leq g\left(t,\left\|T_{0}\right\|\right) \text { where } \\
g \in C\left[\mathbb{R}_{+} \times \mathbb{R}_{+}, \mathbb{R}_{+}\right], g(t, 0)=0 \text {, and the trivial solution of } \\
u^{\prime}=g(t, u), u\left(t_{0}\right)=u_{0} \geq 0
\end{gathered}
$$

is uniformly stable, and

(ii) the trivial solution of (4.1) is uniformly stable.

Then the trivial solution of (4.2) is uniformly stable.

Proof: Any solution $W\left(t, t_{0}, T_{0}\right)$ of (4.2) is of the form

$$
\begin{gathered}
W(t)=Y(t) Y^{-1}\left(t_{0}\right) T_{0} Z^{*^{-1}}\left(t_{0}\right) Z^{*}(t) \\
+Y(t)\left(\int_{t_{0}}^{t} \int_{t_{0}}^{\tau_{1}} \ldots \int_{t_{0}}^{\tau_{n-1}} Y^{-1}(s) F(s, W(s)) Z^{*^{-1}}(s) d s d \tau_{n-1} \ldots d \tau_{1}\right) Z^{*}(t) .
\end{gathered}
$$

If we set $m(t)=\|Y(t) Z(t)\|$ and $\left\|T_{0}\right\|=u_{0}$ with $D^{+} m(t) \leq g(t, m(t))$, then by the comparison theorem [2] we have the estimate

$$
m(t)=\|Y(t) Z(t)\| \leq r\left(t, t_{0}, u_{0}\right)=r\left(t, t_{0},\left\|T_{0}\right\|\right)
$$

the maximal solution of (4.3) with $K=1$. Since $W\left(t_{0}, t_{0}, T_{0}\right)=T\left(t_{0}, t_{0}, T_{0}\right)$, it follows that $\left\|W\left(t, t_{0}, T_{0}\right)\right\|=\| T\left(t, t_{0}, Y(t), Z(t)\|=\| T\left(t, t_{0}, \Phi(t)\right) \|\right.$ where $\Phi$ is a solution of $\Phi^{(n)}=Y^{-1}(t) F(t, \Phi(t)) Z^{*^{-1}}(t)$.

By assumption $(i i)$, given any $\epsilon>0$ and $t_{0}$ in $\mathbb{R}_{+}$, there exists a $\delta(\epsilon)>0$ such that

$$
\left\|T\left(t, t_{0}, T_{0}\right)\right\|<\epsilon \text { whenever } t \geq t_{0} \text { and }\left\|T_{0}\right\|<\delta(\epsilon)
$$


Since the null solution of (4.3) is uniformly stable, we conclude that if $\delta(\epsilon)>0$ for $t_{0} \in \mathbb{R}_{+}$, there exists a $\eta(\epsilon)>0$ such that $r\left(t, t_{0}, u_{0}\right)<\eta(\epsilon)$ whenever $t \geq t_{0}$ if $t_{0}$ is sufficiently small. From (4.4) and (4.5), it follows that $\left\|W\left(t, t_{0}, T_{0}\right)\right\|<\epsilon$ whenever $\left\|T_{0}\right\|<\eta(\epsilon)$ for $t \geq t_{0}$. The proof is complete.

Theorem 4.2: Suppose that

(i) $\quad\left\|Y\left(t, t_{0}, T_{0}\right)\right\| \leq K_{1}$ and $\left\|Y^{-1}\left(t, t_{0}, T_{0}\right)\right\| \leq K_{1}$ for $t \geq t_{0}$ and $\left\|Z\left(t, t_{0}, T_{0}\right)\right\| \leq K_{2}$ and $\left\|Z^{-1}\left(t, t_{0}, T_{0}\right)\right\| \leq K_{2}$ for $t \geq t_{0}$ and $\left\|T_{0}\right\|<\gamma$.

(ii) $\quad\|F(t, W(t))\| \leq g(t,\|W\|)$ where $g \in C\left[\mathbb{R}_{+} \times \mathbb{R}_{+}, \mathbb{R}_{+}\right], g(t, 0)=0, g(t, u)$ is nonincreasing and the trivial solution of

$$
u^{\prime}=K^{2} g(t, u), u\left(t_{0}\right)=u_{0} \geq 0
$$

where $K=K_{1} K_{2}$ is stable. Then the trivial solution of (4.2) is stable.

Proof: Any solution of the perturbed system $T^{\prime}=\sum_{r=0}^{n}\left(\begin{array}{l}n \\ r\end{array}\right) A^{n-r} T(t) B^{r}$ with $B^{0}=A^{0}=I$ is of the form $T(t)=Y(t) C Z^{*}(t)$. This solution satisfies the initial condition $T\left(t_{0}\right)=T_{0}$ if and only if $T(t)=Y(t) Y^{-1}\left(t_{0}\right) T_{0} Z^{-1}\left(t_{0}\right) Z^{*}(t)$. Assumption $(i)$ yields $\|T(t)\| \leq K_{1}^{2} K_{2}^{2}\left\|T_{0}\right\|$ for $\left\|T_{0}\right\|<\gamma$. Suppose there exists a $t_{1} \geq t_{0}$ such that $\|\Phi(t)\|=\left\|Y(t) Y^{-1}\left(t_{0}\right)\right\|<\gamma \quad$ for $\quad t_{0} \leq t<t_{1} \quad$ and $\quad\left\|\Phi\left(t_{1}\right)\right\|=$ $\left\|Y\left(t_{1}\right) Y^{-1}\left(t_{0}\right)\right\|=\gamma$. Then any solution of (4.2) is of the form

$$
\begin{gathered}
W(t)=Y(t) Y^{-1}\left(t_{0}\right) T_{0} Z^{*^{-1}}\left(t_{0}\right) Z^{*}(t) \\
+Y(t)\left(\int_{t_{0}}^{t} \int_{t_{0}}^{\tau_{1}} \ldots \int_{t_{0}}^{\tau_{n-1}} Y^{-1}(s) F(s, W(s)) Z^{*^{-1}}(s) d s d \tau_{n-1} \ldots d \tau_{1}\right) Z^{*}(t) .
\end{gathered}
$$

Using the bounds on $Y(t), Z(t), Y^{-1}(t)$, and $Z^{-1}(t)$ along with assumption $(i i)$, we get

$$
\|W(t)\| \leq K_{1}^{2} K_{2}^{2}\left\|T_{0}\right\|+K_{1}^{2} K_{2}^{2} \int_{t_{0}}^{t} \int_{t_{0}}^{\tau_{1}} \ldots \int_{t_{0}}^{\tau_{n-1}} g(s,\|W(s)\|) d s d \tau_{n-1} \ldots d \tau_{1} .
$$

Hence,

$$
\|W(t)\| \leq K^{2}\left\|T_{0}\right\|+K^{2} \int_{t_{0}}^{t} \int_{t_{0}}^{t} \ldots \int_{t_{0}}^{\tau_{1}} g(s,\|W(s)\|) d s d \tau_{n-1} \ldots d \tau_{1} .
$$

This fact leads to

$$
\|W(t)\| \leq r\left(t, t_{0}, u_{0}\right)=r\left(t, t_{0},\left\|T_{0}\right\|\right),
$$

for $t_{0} \leq t<t_{1}$ where $r\left(t, t_{0}, u_{0}\right)$ is the maximal solution of $u^{\prime}=K^{2} g(t, u), u\left(t_{0}\right)=u_{0}$ such that $\left\|T_{0}\right\| \leq u_{0}$. By using (ii), given $\eta>0$, there exists a $\delta_{1}>0$ such that $r\left(t, t_{0}, u_{0}\right)<\eta$ for $t \geq t_{0}$ whenever $u_{0}<\delta_{1}$. Thus, if $\left\|T_{0}\right\| \leq u_{0}<\delta_{1}$, then $\|W(t)\|<\eta$ for all $t \geq t_{0}$. This contradicts the fact that $\left\|\Phi\left(t_{1}\right)\right\|=\left\|Y\left(t_{1}\right) Y^{-1}\left(t_{0}\right)\right\|=\eta$. Thus, $\left\|\Phi\left(t_{1}\right)\right\|<\eta$ for all $t \geq t_{0}$. Now,

$$
\|W(t)\| \leq K_{1}^{2} K_{2}^{2}\left\|T_{0}\right\|+K_{1}^{2} K_{2}^{2} \int_{t_{0}}^{t} \int_{t_{0}}^{\tau_{1}} \ldots \int_{t_{0}}^{\tau_{n-1}} g(s,\|W(s)\|) d s d \tau_{n-1} \ldots d \tau_{1} .
$$

Thus, $\left\|W\left(t, t_{0}, T_{0}\right)\right\| \leq r\left(t, t_{0}, u_{0}\right)$ where $r(t)$ is the maximal solution of (4.6). Given any $\epsilon>0$, there exists a $\delta=\delta\left(\epsilon, t_{0}\right)>0$ such that $\left\|T_{0}\right\|<\delta$ implies

$$
\|W(t)\| \leq r\left(t, t_{0}, u_{0}\right)=r\left(t, t_{0},\left\|T_{0}\right\|\right)<\epsilon
$$


for all $t \geq t_{0}$. Thus, the null solution of (4.2) is stable.

The following theorem gives a set of sufficient conditions for the uniform Lipschitz stability of the perturbed matrix differential system (4.2).

Theorem 4.3: Suppose that

(i) the zero solution of the unperturbed system (4.1) is uniformly Lipschitz stable and that hypothesis $(i)$ of Theorem 4.2 holds.

(ii) $\quad \| F\left(t, W(t)\|\leq \gamma(t)\| W \|\right.$ where $\gamma \in C\left[\mathbb{R}_{+}, \mathbb{R}\right]$ and

$$
\int_{t_{0}}^{t} \int_{t_{0}}^{\tau_{1}} \ldots \int_{t_{0}}^{\tau_{n-1}} \gamma(s) d s d \tau_{n-1} \ldots d \tau_{1}<\infty \text { for all } t \geq t_{0} .
$$

Then the zero solution of (4.2) is uniformly Lipschitz stable.

Proof: Let $T\left(t, t_{0}, T_{0}\right)$ be the solution of (4.1) and $W\left(t, t_{0}, T_{0}\right)$ be the solution of the perturbed system (4.2). Then clearly

$$
\begin{aligned}
& \left\|T\left(t, t_{0}, T_{0}\right)\right\| \leq\left\|W\left(t, t_{0}, T_{0}\right)\right\|=\| Y(t) Y^{-1}\left(t_{0}\right) T_{0} Z^{*^{-1}}\left(t_{0}\right) Z^{*}(t) \\
& +Y(t)\left(\int_{t_{0}}^{t} \int_{t_{0}}^{\tau_{1}} \ldots \int_{t_{0}}^{\tau_{n-1}} Y^{-1}(s) F(s, W(s)) Z^{*^{-1}}(s) d s d \tau_{n-1} \ldots d \tau_{1}\right) Z^{*}(t) \| \\
& \leq K_{1}^{2} K_{2}^{2}\left\|T_{0}\right\|+K_{1}^{2} K_{2}^{2} \int_{t_{0}}^{t} \int_{t_{0}}^{\tau_{1}} \ldots \int_{t_{0}}^{\tau_{n-1}} \gamma(s)\|W(s)\| d s d \tau_{n-1} \ldots d \tau_{1} .
\end{aligned}
$$

By the Gronwall-Reid-Bellman inequality

$$
\begin{gathered}
\left\|W\left(t, t_{0}, T_{0}\right)\right\| \leq K_{1}^{2} K_{2}^{2}\left\|T_{0}\right\| \exp \left(K_{1}^{2} K_{2}^{2}\right) \int_{t_{0}}^{t} \int_{t_{0}}^{\tau_{1}} \ldots \int_{t_{0}}^{\tau_{n-1}} \gamma(s) d s d \tau_{n-1} \ldots d \tau_{1} \\
\leq K_{1}^{2} K_{2}^{2}\left\|T_{0}\right\| \exp \left(K_{1}^{2} K_{2}^{2}\right) \int_{t_{0}}^{\infty} \int_{t_{0}}^{\tau_{1}} \ldots \int_{t_{0}}^{\tau_{n-1}} \gamma(s) d s d \tau_{n-1} \ldots d \tau_{1} \leq L\left\|T_{0}\right\|,
\end{gathered}
$$

where $L=K_{1}^{2} K_{2}^{2} \exp \left(K_{1}^{2} K_{2}^{2}\right) \int_{t_{0}}^{\infty} \int_{t_{0}}^{\tau_{1}} \ldots \int_{t_{0}}^{\tau_{n-1}} \gamma(s) d s d \tau_{n-1} \ldots d \tau_{1}$. Thus, the proof is complete.

\section{Controllability and Observability}

Consider the Lyapunov system

$$
\begin{gathered}
T^{(n)}=A^{n} T(t)+n C_{1} A^{n-1} T(t) B+n C_{2} A^{n-2} T(t) B^{2}+\ldots+n C_{r} A^{n-r} T(t) B^{r}+\ldots \\
+T(t) B^{n}+C(t) U(t) D^{*}(t)
\end{gathered}
$$

$W(t)=G(t) T(t) H^{*}(t)$ where $A, B, T$ are all square matrices of order $n \times n$. $C$ and $D$ are $n \times m$ matrices and $U$ is an $m \times m$ matrix. We call $U$ the control and $W$ the observation.

Definition 5.1: The system (5.1) is said to be completely controllable if for any given initial condition $T\left(t_{0}\right)=T_{0}$ and for any $t_{1} \geq t_{0}$, there is some $U(t)$ defined on $t_{0} \leq t \leq t_{1}$ such that $T(t)=0$.

Definition 5.2: The system (5.1) is said to be observable if for any given $t_{1} \geq t_{0}$ we can determine $T(t)$ for $t_{0} \leq t \leq t_{1}$ from $W(t)$. 
From the variation of parameters formula, the solution of system (2.4) has the form

$T(t)=Y(t) C Z^{*}(t)$

$$
+Y(t)\left(\int_{a}^{t} \int_{a}^{\tau_{1}} \int_{a}^{\tau_{2}} \ldots \int_{a}^{\tau_{n-1}} Y^{-1}(s) F(s, T(s)) Z^{*^{-1}}(s) d s d \tau_{n-1} \ldots d \tau_{2} d \tau_{1}\right) Z^{*}(t) .
$$

Then,

$$
\begin{aligned}
& Y^{-1}(t) T(t) Z^{*^{-1}}(t) \\
& =C+\left(\int_{a}^{t} \int_{a}^{\tau_{1}} \int_{a}^{\tau_{2}} \ldots \int_{a}^{\tau_{n-1}} Y^{-1}(s) F(s, T(s)) Z^{*^{-1}}(s) d s d \tau_{n-1} \ldots d \tau_{2} d \tau_{1}\right)
\end{aligned}
$$

and

$$
\begin{aligned}
C=Y^{-1}(t) & T(t) Z^{*^{-1}}(t) \\
& \quad-\left(\int_{a}^{t} \int_{a}^{\tau_{1}} \int_{a}^{\tau_{2}} \ldots \int_{a}^{\tau_{n-1}} Y^{-1}(s) F(s, T(s)) Z^{*^{-1}}(s) d s d \tau_{n-1} \ldots d \tau_{2} d \tau_{1}\right) .
\end{aligned}
$$

From this, we can determine $C$ and apply the variation of parameters formula to determine $T(t)$.

Theorem 5.1: Suppose for some $t_{1} \geq t_{0}$ the matrices $G^{*} Y^{*} Y$ and $H Z Z^{*} H^{*}$ are positive definite. Then the system (5.1) is observable.

Proof: Let $W(t)=G(t) T(t) H(t)$. Then

$$
Y\left(t_{1}\right) W\left(t_{1}\right) Z^{*}\left(t_{1}\right)=Y\left(t_{1}\right) G\left(t_{1}\right) T\left(t_{1}\right) H\left(t_{1}\right) Z^{*}\left(t_{1}\right)
$$

and hence

$$
\begin{gathered}
G^{*}\left(t_{1}\right) Y^{*}\left(t_{1}\right) Y\left(t_{1}\right) W\left(t_{1}\right) Z^{*}\left(t_{1}\right) Z\left(t_{1}\right) H\left(t_{1}\right) \\
=G^{*}\left(t_{1}\right) Y^{*}\left(t_{1}\right) Y\left(t_{1}\right) G\left(t_{1}\right) T\left(t_{1}\right) H\left(t_{1}\right) Z^{*}\left(t_{1}\right) Z\left(t_{1}\right) H\left(t_{1}\right) .
\end{gathered}
$$

Since the matrices $G^{*} Y^{*} Y$ and $H Z Z^{*} H^{*}$ are positive definite, whenever $G$ and $H$ are nonsingular, we can solve for $T\left(t_{1}\right)$ and hence for $T(t)$ for $t \geq t_{0}$.

Theorem 5.2: If $Y^{-1}(t) C(t) C^{*}(t) Y^{*^{-1}}(t)$ and $Y^{-1}(t) D(t) D^{*}(t) Z^{*^{-1}}(t)$ are all positive definite for all $t$, then the matrix system is completely controllable.

Proof: Any solution $T(t)$ of (5.1) is of the form

$T(t)=Y(t) C Z^{*}(t)$

$$
+Y(t)\left(\int_{a}^{t} \int_{a}^{\tau_{1}} \int_{a}^{\tau_{2}} \ldots \int_{a}^{\tau_{n-1}} Y^{-1}(s) C(s) U(s) D^{*}(s) Z^{*^{-1}}(s) d s d \tau_{n-1} \ldots d \tau_{2} d \tau_{1}\right) Z^{*}(t) \text {.If we }
$$

choose

$$
\left.C=-\int_{a}^{t} \int_{a}^{\tau_{1}} \int_{a}^{\tau_{2}} \ldots \int_{a}^{\tau_{n-1}} Y^{-1}(s) C(s) U(s) D^{*}(s) Z^{*^{-1}}(s) d s d \tau_{n-1} \ldots d \tau_{2} d \tau_{1}\right) Z^{*}(t),
$$

then $T(t)=0$. Choose $U$ as 


$$
\begin{aligned}
U(t)= & -\frac{n !}{\left(t_{1}-t_{0}\right)^{n}} C^{*}(t) Y^{*^{-1}}(t)\left\{Y^{-1}(t) C(t) C^{*}(t) Y^{*^{-1}}(t)\right\}^{-1} C \\
& \times\left\{Z^{-1}(t) D(t) D^{*}(t) Z^{*^{-1}}(t)\right\}^{-1} Z^{-1}(t) D(t) .
\end{aligned}
$$

If we substitute this expression into (5.1), we find that the equality holds. Thus, the system (5.1) is completely controllable.

\section{References}

[1] Bellman, R., Stability Theory of Differential Equations, McGraw-Hill, New York 1953.

[2] Fausett, D.F. and Köksal, S., Variation of parameters formula and Lipschitz stability of nonlinear matrix differential equations, Proc. of the First World Congress of Nonlinear Analysts, Walter deGruyter, Berlin 1996.

[3] Lakshmikantham, V. and Deo, S.G., Method of Variation of Parameters for Dynamic Systems, Gordon and Breach Science Publishers, UK 1998.

[4] Murty, K.N., Howell, G. and Sivasundaram, S., Two (multi) point nonlinear Lyapunov systems existence and uniqueness, JMAA 167 (1992), 505-512. 


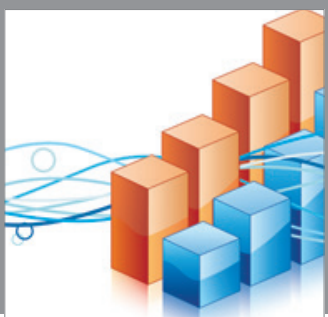

Advances in

Operations Research

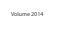

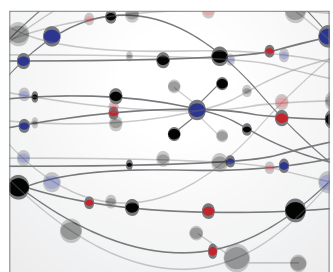

\section{The Scientific} World Journal
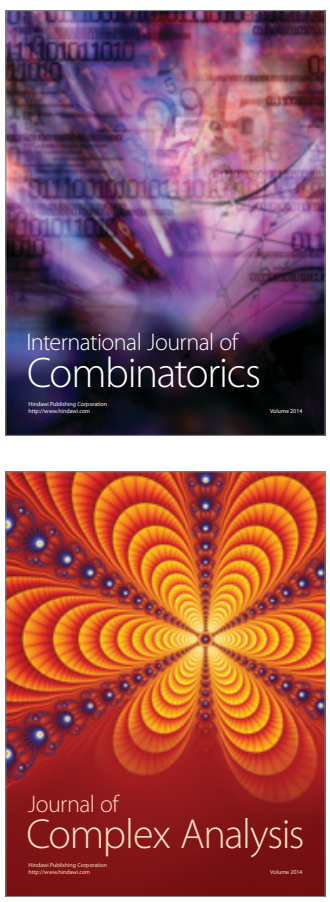

International Journal of

Mathematics and

Mathematical

Sciences
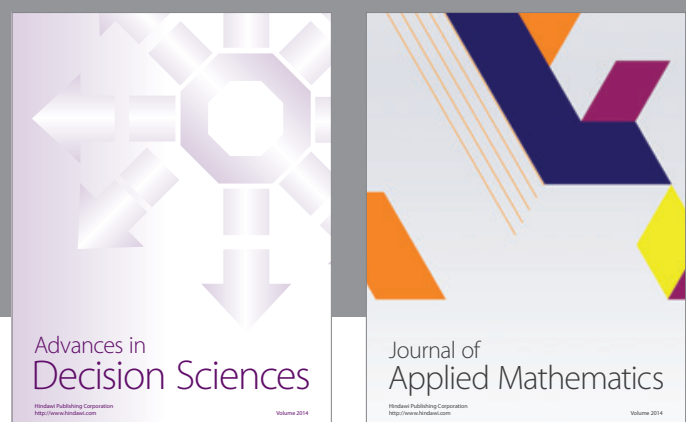

Journal of

Applied Mathematics
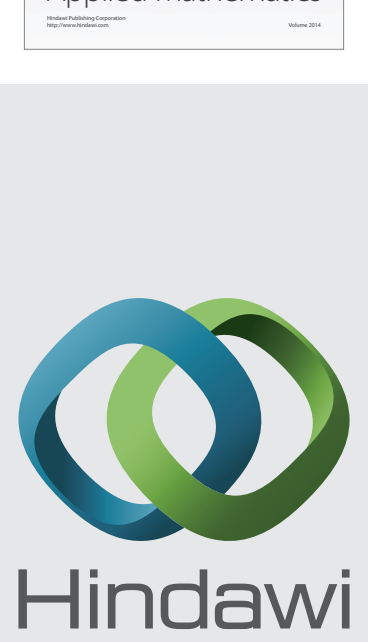

Submit your manuscripts at http://www.hindawi.com
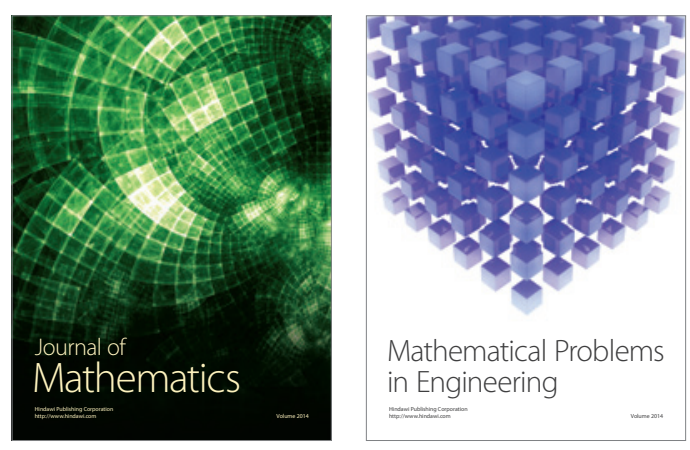

Mathematical Problems in Engineering
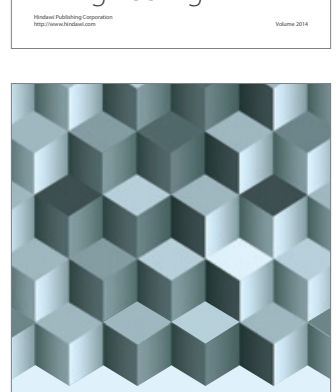

Journal of

Function Spaces
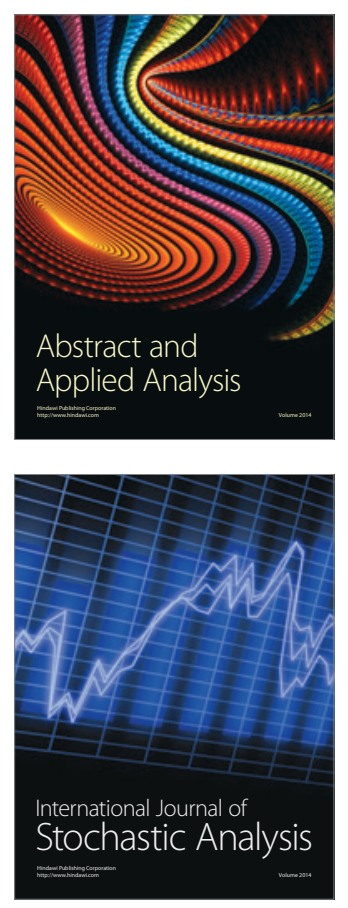

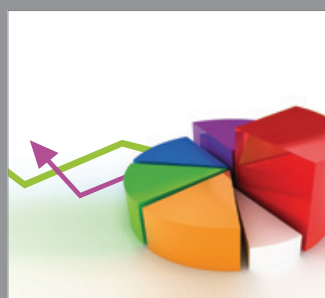

ournal of

Probability and Statistics

Promensencen
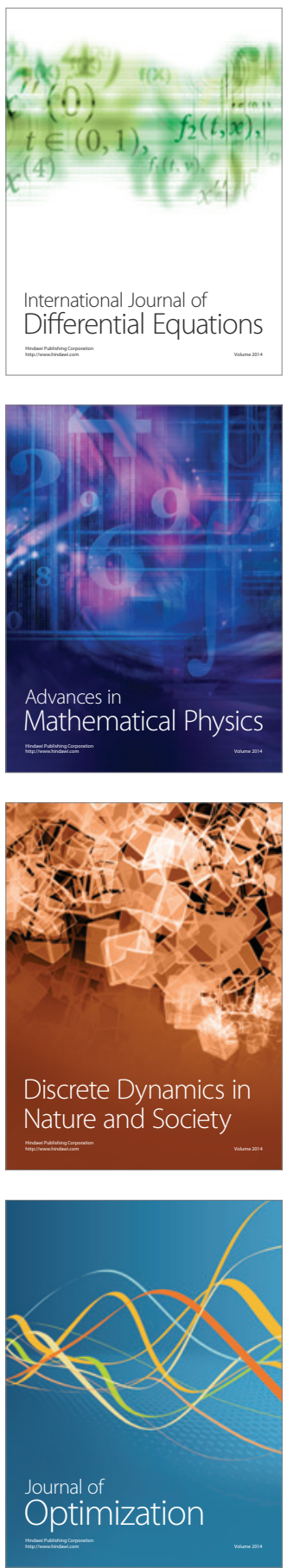